\title{
Channel Adaptive Encoding and Decoding Strategies and Rate Regions for the Three User Cooperative Multiple Access Channel
}

\author{
Çag̃atay Edemen Onur Kaya \\ Department of Electronics Engineering \\ Işık University \\ Şile, Istanbul, Turkey \\ cagatay.edemen@isik.edu.tr_onurkaya@isikun.edu.tr
}

\begin{abstract}
For a cooperative Gaussian multiple access channel (MAC), we propose a new channel adaptive three user cooperation strategy, based on a non-trivial extension of block Markov superposition encoding. We obtain the expressions for the resulting achievable rate region. We demonstrate through simulations that the participation of an extra user in cooperation provides significant rate improvements. The proposed strategy also improves upon our earlier results on the three user cooperative MAC [1], under certain channel conditions.
\end{abstract}

\section{INTRODUCTION}

The increasing demand for higher rates in wireless communication systems calls for the development of new communication strategies which make efficient use of the available resources. User cooperation is a very good example of such strategies, in that it exploits what comes for free in wireless networks, side information, to create additional diversity in transmissions, by forming a virtual antenna array for transmissions.

The idea of mutual user cooperation in wireless networks roots from the pioneering works of Carleial [2] and Willems et al. [3] on multiple access channels with generalized feedback (MAC-GF), which is a very suitable model for wireless channels as it takes into account the over-heard information by the transmitters. More recently, Sendonaris et al. [4] applied the results of [3] to obtain the achievable rates for a cooperative Gaussian MAC in the presence of fading. Since then, there has been an increasing amount of interest in cooperative communication, and numerous new results on its various aspects were obtained. An extensive set of references on cooperative communications can be found in [5].

The extension of cooperative strategies from two to multiple users has also been widely investigated, but the main focus has been on strategies relying on dedicated relaying rather than mutual cooperation. One such line of work is the multiple relay channel, which is composed of a single transmitter and many relays [6], [7], [8], [9], [10]. A second line of work is the multiple-access relay channel, which is composed of an $\mathrm{M}$-user MAC, and one additional relay whose sole task is to assist the MAC users in their transmission [11], [12]. However, systems where all participating users mutually cooperate have not received much attention, perhaps due to the difficulty of generalizing the encoding strategies, and more importantly, characterizing the seemingly much more complicated achievable rate regions.

In this paper, we focus on the relatively untouched three user Gaussian cooperative MAC, which contains as special cases the

This work was supported by The Scientific \& Technological Research Council of Turkey, Grant 106E018.

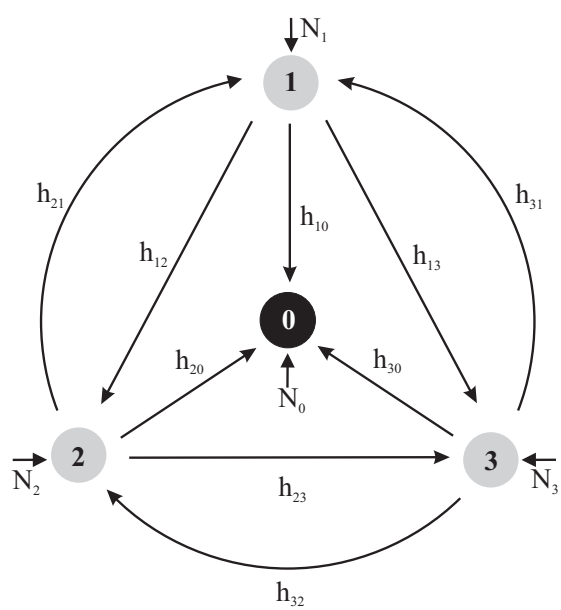

Fig. 1. Three user cooperative channel model

multiple relay channel and the multiple access relay channel. In a related recent work, we have obtained some preliminary results on non-trivial extensions of the block Markov encoding policy to three users, and the associated achievable rate regions, for a certain ordering of the channel states of the users [1]. This paper is intended as a sequel to [1], aiming to complete the characterization of the channel adaptive encoding and decoding policies, for all possible orderings of the receive-link qualities at the cooperating users. We first propose a new channel adaptive decoding strategy at the transmitters for building up the cooperative information, and then we extend the block Markov superposition encoding for the 2 user MAC-GF to three users. The resulting encoding and decoding strategy differs from that in [1] in terms of the structure of the cooperative codewords, and it is shown to outperform the strategy proposed in [1], even in some cases where the channel ordering does not seem in favor of suggesting the use of the new strategy. We provide expressions for the achievable rates and a 3-D achievable rate region obtained by evaluating those expressions.

\section{SYSTEM MODEL}

We consider a three user fading Gaussian MAC, where both the receiver and the transmitters receive noisy versions of the transmitted messages, as illustrated in Figure 1. The transmitters are assumed to be operating in the full duplex mode. The system is modelled by,

$$
\begin{aligned}
& Y_{0}=\sqrt{h_{10}} X_{1}+\sqrt{h_{20}} X_{2}+\sqrt{h_{30}} X_{3}+N_{0} \\
& Y_{1}=\sqrt{h_{21}} X_{2}+\sqrt{h_{31}} X_{3}+N_{1} \\
& Y_{2}=\sqrt{h_{12}} X_{1}+\sqrt{h_{32}} X_{3}+N_{2} \\
& Y_{3}=\sqrt{h_{13}} X_{1}+\sqrt{h_{23}} X_{2}+N_{3}
\end{aligned}
$$


where $X_{i}$ is the symbol transmitted by node $i, Y_{i}$ is the symbol received at node $i$, and the receiver is denoted by $i=0 ; N_{i}$ is the zero-mean additive white Gaussian noise at node $i$, having variance $\sigma_{i}^{2}$, and $\sqrt{h_{i j}}$ are the random fading coefficients, the instantaneous realizations of which are assumed to be known by both the transmitters and the receiver. We further define the normalized fading coefficients $s_{i j}=\frac{h_{i j}}{\sigma_{j}^{2}}$, for the simplicity of our discussions.

Throughout this paper, we assume that the normalized channel gains satisfy $s_{i j}>s_{i 0}, \forall i, j \in\{1,2,3\}, i \neq j$; that is, the inter-user cooperation links are uniformly stronger than the direct links. This particular case is of practical interest since the cooperating transmitters are likely to be closely located with less number of scatterers and obstructions on the paths connecting them, when compared to their paths to the receiver, and thus have better channel conditions among each other.

\section{AN EXTENSION OF BLOCK MARKov ENCODING}

For a multiple access channel with generalized feedback, the extension of the block Markov encoding policy from the two user channel to three users is non-trivial, since the cooperation options become more diverse, as the number of cooperating users increases.

For the two user MAC-GF, the encoding is performed by dividing each user's message $w_{i}$ into two sub-messages, one used solely to introduce fresh information intended for the receiver, and the other used for simultaneously transmitting cooperative information to both the cooperating partner and the receiver [3], [4]. For a three user MAC-GF, it is natural to extend this strategy by simply including additional cooperative sub-messages intended for each user from each transmitter, i.e.,

$$
w_{i}=\left(w_{i 0}, w_{i j}, w_{i k}\right), \quad i \neq j \neq k, \quad i, j, k \in\{1,2,3\}
$$

However, with the introduction of new sub-messages, one has to decide on how to encode these messages into cooperative codewords, i.e., which messages should be used by which users for cooperation. This also requires a decision about which cooperative messages should be decoded by which receivers. If the user $i$ were to decode only the messages $w_{j i}$ solely intended for itself, treating all other signals as noise as it is done in the two user MAC-GF in [4], it would face a significant amount of interference in reception over the inter-user links. This would eventually degrade the quality of the inter-user links and possibly reduce the rate advantage due to the cooperation among the users. In order to control the interference over the inter user links, we have proposed in [1] an extension of the block Markov coding strategy, based on relative receive-link qualities for the users. The encoding and decoding strategies in [1] were limited in the sense that they were designed only for a specific ordering of the inter-user links,

$$
s_{i j}>s_{i k}, \quad s_{j i}>s_{j k}, \quad s_{k j}>s_{k i}, \quad i \neq j \neq k
$$

that lead to user $j$ being the strongest, and decoding all the information sent over the channel, and user $k$ being the weakest, and decoding only the information intended for itself. The resulting decoding strategies for each participating transmitter, which we will call policy I in this paper, are summarized in
TABLE I

DECODING STRATEGY AT THE TRANSMITTERS: POLICY I [1]

\begin{tabular}{|l|l|l|}
\hline User & Decoded Messages & Own Messages \\
\hline \hline $\mathrm{i}$ & $w_{j i}, w_{k i}, w_{j k}$ & $w_{i j}, w_{i k}$ \\
$\mathrm{j}$ & $w_{i j}, w_{k j}, w_{i k}, w_{k i}$ & $w_{j i}, w_{j k}$ \\
$\mathrm{k}$ & $w_{i k}, w_{j k}$ & $w_{k i}, w_{k j}$ \\
\hline
\end{tabular}

TABLE II

DECODING STRATEGY AT THE TRANSMITTERS: POLICY II

\begin{tabular}{|l|l|l|}
\hline User & Decoded Messages & Own Messages \\
\hline \hline $\mathrm{i}$ & $w_{j i}, w_{k i}, w_{k j}$ & $w_{i j}, w_{i k}$ \\
$\mathrm{j}$ & $w_{i j}, w_{i k}, w_{k j}$ & $w_{j i}, w_{j k}$ \\
$\mathrm{k}$ & $w_{i k}, w_{j i}, w_{j k}$ & $w_{k i}, w_{k j}$ \\
\hline
\end{tabular}

Table I. In this paper, we introduce a new encoding/decoding policy, which is designed for the remaining possible orderings of the instantaneous channel states, i.e.,

$$
s_{i j}>s_{i k}, \quad s_{j k}>s_{j i}, \quad s_{k i}>s_{k j}, \quad i \neq j \neq k
$$

As in [1], our proposed encoding and decoding strategy is inspired by the capacity achieving encoding/decoding for Gaussian broadcast channels, where the stronger receiver decodes not only its own message, but also the weaker users' messages.

It is easy to check that, there are a total of eight possible orderings for the receive-link qualities at the users, six of which obey (6), and two of which obey (7). Unlike the asymmetric situation caused by ordering (6), when the channel qualities satisfy (7), each user has better reception quality on one of the underlying broadcast channels, and worse on the other. For the simplicity of the exposition, we will assume from now on without loss of generality that

$$
s_{12}>s_{13}, \quad s_{23}>s_{21}, \quad s_{31}>s_{32}
$$

Based on this assumption, user 2 has the stronger receivelink for the transmission of user 1 . If user 1 were broadcasting alone, user 2 would be able to correctly decode not only its own intended message $w_{12}$, but also the message $w_{13}$ intended for user 3 , provided message $w_{13}$ was being transmitted at a rate that is supported at user 3 . The same argument holds for all other broadcast scenarios, in each of which only a distinct user is the stronger one. Motivated by these observations, we propose a variation of the decoding policies for the transmitters suited for the ordering in (7). This new decoding policy, which we call policy II, is summarized in Table II. The decoding policy for the specific ordering in (8) is then simply obtained by substituting $i=1, j=2, k=3$ in Table II.

Although the derivation of decoding strategies closely followed the ideas in [1], the structure of the resulting cooperation signals are significantly different. From Table II, one can observe that the messages $w_{13}, w_{21}$ and $w_{32}$ are known to all transmitters, but there are no pairs of messages known to more than one transmitter. The message $w_{12}$ is only known to the transmitters 1 and $2 ; w_{23}$ only to 2 and 3 , and $w_{31}$ only to 1 and 3 . This grouping of common information calls for the following new way to form the cooperation signals. We use one cooperation signal common to all users, which is a function of three sub-messages, and three other cooperation signals common to each pair of users, which are functions of just one sub-message 
TABLE III

CODEBOOK GENERATION AND ENCODING AT THE TRANSMITTERS

\begin{tabular}{|l|l|}
\hline User & Codewords \\
\hline \hline 1 & $U\left(w_{13}^{\prime}, w_{21}^{\prime}, w_{32}^{\prime}\right), U_{1}\left(w_{12}^{\prime}, U\right), U_{3}\left(w_{31}^{\prime}, U\right)$, \\
& $X_{12}\left(w_{12}, U_{1}, U\right), X_{13}\left(w_{13}, U_{3}, U\right)$, \\
& $X_{10}\left(w_{10}, X_{12}, X_{13}, U_{1}, U_{3}, U\right)$ \\
\hline 2 & $U\left(w_{13}^{\prime}, w_{21}^{\prime}, w_{32}^{\prime}\right), U_{1}\left(w_{12}^{\prime}, U\right), U_{2}\left(w_{23}^{\prime}, U\right)$, \\
& $X_{21}\left(w_{21}, U_{1}, U\right), X_{23}\left(w_{23}, U_{2}, U\right)$, \\
& $X_{20}\left(w_{20}, X_{21}, X_{23}, U_{1}, U_{2}, U\right)$ \\
\hline 3 & $U\left(w_{13}^{\prime}, w_{21}^{\prime}, w_{32}^{\prime}\right), U_{3}\left(w_{31}^{\prime}, U\right), U_{2}\left(w_{23}^{\prime}, U\right)$, \\
& $X_{31}\left(w_{31}, U_{3}, U\right), X_{32}\left(w_{32}, U_{2}, U\right)$, \\
& $X_{30}\left(w_{30}, X_{31}, X_{32}, U_{2}, U_{3}, U\right)$ \\
\hline
\end{tabular}

each, a little reminiscent of the coding for the relay channel. By a suitable extension of the codebook generation process described in [3], [4], we perform the codebook generation and encoding as summarized in Table III. In Table III, the submessages $w_{i j}^{\prime}$ denote the messages received in the previous block: the cooperation signals depend on the messages received in previous block, and new information is also encoded into codewords $X_{i j}$, taking into account the messages received in the previous block. The order in the codebook generation is also observed in Table III: the collective cooperation signals $U$ are generated first, then the pairwise cooperation signals, and so on.

Then, the signals transmitted by each user can be generated by block Markov superposition encoding as follows:

$$
\begin{aligned}
X_{1}= & \sqrt{P_{10}} X_{10}+\sqrt{P_{12}} X_{12}+\sqrt{P_{13}} X_{13} \\
& +\sqrt{P_{1 U_{1}}} U_{1}+\sqrt{P_{1 U_{3}}} U_{3}+\sqrt{P_{1 U}} U \\
X_{2}= & \sqrt{P_{20}} X_{20}+\sqrt{P_{21}} X_{21}+\sqrt{P_{23}} X_{23} \\
& +\sqrt{P_{2 U_{1}}} U_{1}+\sqrt{P_{2 U_{2}}} U_{2}+\sqrt{P_{1 U}} U \\
X_{3}= & \sqrt{P_{30}} X_{30}+\sqrt{P_{31}} X_{31}+\sqrt{P_{32}} X_{32} \\
& +\sqrt{P_{3 U_{2}}} U_{2}+\sqrt{P_{3 U_{3}}} U_{3}+\sqrt{P_{3 U}} U
\end{aligned}
$$

Here, the codewords $X_{i 0}$ carry the fresh information intended for the receiver, $X_{i j}$ carry the information intended for transmitter $j$ for cooperation in the next block. The cooperation codeword $U$ carries the common information sent by all three users; and the cooperation codewords $U_{1}, U_{2}$ and $U_{3}$ relay the sub-messages common to each pair of users, for the resolution of the remaining uncertainty from the previous block. All codewords are chosen from unit-power Gaussian distributions. The transmit powers are then captured by the powers associated with each component, which are required to satisfy the average power constraints,

$$
\begin{aligned}
& P_{10}+P_{12}+P_{13}+P_{1 U_{1}}+P_{1 U_{3}}+P_{1 U} \leq P_{1} \\
& P_{20}+P_{21}+P_{23}+P_{2 U_{1}}+P_{2 U_{2}}+P_{2 U} \leq P_{2} \\
& P_{30}+P_{31}+P_{32}+P_{3 U_{2}}+P_{3 U_{3}}+P_{3 U} \leq P_{3}
\end{aligned}
$$

Note that, encoding and decoding policies I and II described in Tables I and II respectively are sufficient to cover all possible channel state orderings, and can be used adaptively based on the channel state information, to maximize the rates. Therefore, the proposed policy in this paper complements the policy of [1], thereby yielding a channel adaptive three user block Markov encoding policy.

\section{The Achievable Rate Region}

Before proceeding to characterize the rate region, we make one further simplification to the encoding policy. In [13], it has been shown that when the cooperative links are stronger than the direct links, the optimum strategy of the users is to send only cooperative information, and discard $X_{i 0}$. Although in order to prove a similar result for the three user cooperative $\mathrm{MAC}$, the rate regions need to be established, and then power optimized for the general case; we simply choose to assume that the cooperative links are uniformly stronger than the direct links of the users, and drop the codewords $X_{i 0}$ from our encoding policy, so that the rate regions are easier to obtain and simulate.

The rate constraints bounding the achievable rate region are easiest viewed in two groups: those necessary for reliable decoding at the transmitters, and those necessary for reliable decoding at the ultimate receiver.

As far as the reliable decoding at the transmitters is concerned, it is easy to see that for each transmitter we have a multiple access channel with a group of independent messages that need to be decoded, and an extra message which will be treated as noise. Classical arguments on capacity regions for multiple access channels [14] can be used to obtain the following rate constraints, corresponding to the decodings at users 1,2 and 3 respectively.

$$
\begin{aligned}
\sum_{\{i, j\} \in \Gamma_{1}} R_{i j}<E\left[\log \left(1+\frac{\sum_{\{i, j\} \in \Gamma_{1}} s_{i 1} P_{i j}}{A}\right)\right] \\
\sum_{\{i, j\} \in \Gamma_{2}} R_{i j}<E\left[\log \left(1+\frac{\sum_{\{i, j\} \in \Gamma_{2}} s_{i 2} P_{i j}}{B}\right)\right] \\
\sum_{\{i, j\} \in \Gamma_{3}} R_{i j}<E\left[\log \left(1+\frac{\sum_{\{i, j\} \in \Gamma_{3}} s_{i 3} P_{i j}}{C}\right)\right] \\
\forall \Gamma_{1} \subset\{\{2,1\},\{3,1\},\{3,2\}\}, \\
\forall \Gamma_{2} \subset\{\{1,2\},\{1,3\},\{3,2\}\}, \\
\forall \Gamma_{3} \subset\{\{1,3\},\{2,1\},\{2,3\}\} .
\end{aligned}
$$

Here, the interference plus noise terms $A, B, C$ are defined as,

$$
\begin{aligned}
& A=1+s_{21}\left(P_{23}+P_{2 U_{2}}\right)+s_{31} P_{3 U_{2}}+2 \sqrt{s_{21} s_{31} P_{2 U_{2}} P_{3 U_{2}}} \\
& B=1+s_{12} P_{1 U_{3}}+s_{32}\left(P_{31}+P_{3 U_{3}}\right)+2 \sqrt{s_{12} s_{32} P_{1 U_{3}} P_{3 U_{3}}} \\
& C=1+s_{13}\left(P_{12}+P_{1 U_{1}}\right)+s_{23} P_{2 U_{1}}+2 \sqrt{s_{13} s_{23} P_{1 U_{1}} P_{2 U_{1}}}
\end{aligned}
$$

It is interesting to note that the users suffer from the effect of coherent combining in the interference terms. The major difference from the rate region in [1] is that, the rate constraints are now symmetric, and the rates of user 3 are now less prone to interference, whereas there is some added interference at user 2, due to the decoding assumption. In the simulation results section, we will demonstrate that sometimes the more symmetric policy II may in fact produce better achievable rates, even for channel states it is not designed for, i.e., those 
satisfying (6), which justifies the novelty and usefulness of policy II proposed in this paper.

The rate constraints for error free decoding at the receiver are also obtained by using capacity results for the traditional MAC. However, one has to take into account the effect of backwards decoding: in a given block, the receiver first decodes the cooperative information, which consists of sub-messages encoded in groups into codewords $U, U_{1}, U_{2}$ and $U_{3}$. Therefore, the submessages $w_{13}, w_{21}$ and $w_{32}$, should be treated as one single message and should be jointly decoded. Keeping this in mind, the rate constraints that need to be satisfied at the receiver are obtained as given in equations (19)-(26), where,

$$
\begin{aligned}
D= & 2 \sqrt{s_{10} s_{20} P_{1 U 1} P_{2 U 1}} \\
E= & 2 \sqrt{s_{10} s_{30} P_{1 U 3} P_{3 U 3}} \\
F== & 2 \sqrt{s_{20} s_{30} P_{2 U 2} P_{3 U 2}} \\
G= & 2\left(\sqrt{s_{10} s_{20} P_{1 U} P_{2 U}}+\sqrt{s_{10} s_{30} P_{1 U} P_{3 U}}\right. \\
& \left.+\sqrt{s_{20} s_{30} P_{2 U} P_{3 U}}\right)
\end{aligned}
$$

Then, the overall achievable rate region is found as the convex hull of rate triplets $R_{1}=R_{12}+R_{13}, R_{2}=R_{21}+R_{23}$, $R_{3}=R_{31}+R_{32}$ satisfying (12)-(26).

\section{Simulation Results}

In this section, we demonstrate the usefulness of the proposed three user cooperation strategy by evaluating the achievable rate region for several fading scenarios, and comparing it to the corresponding two user cooperative system, as well as the encoding/decoding policy proposed in [1].

We first evaluate the rate region achievable by policy II, for a fading distribution which satisfies the assumption in (8), that is, $s_{10}, s_{20}, s_{30}$ are i.i.d uniform random variables taking the values from the set $\{0.1: 0.2: 0.9\}, s_{13}, s_{21}, s_{32}$ are i.i.d taking values from $\{1.1: 0.2: 1.9\}$ and $s_{12}, s_{23}, s_{31}$ are also i.i.d with values $\{2.1: 0.2: 2.9\}$. The average transmit power for each user is chosen to be 1 in all simulations in this section. The 3-D achievable rate region is plotted in Figure 2 (outer region), along with the 2-D two user cooperative MAC achievable rate regions [4] (inner regions only on $R_{i}-R_{j}$ planes), evaluated for the same fading distributions. There are two important observations: firstly, the presence of the third user improves the achievable rates significantly, when compared to the two user strategy: simply compare the two strategies on the planes corresponding to $R_{i}=0$. Secondly, the maximum values for individual rates are asymmetric for the
TABLE IV

FADING DISTRIBUTIONS SATISFYING (8)

\begin{tabular}{|l|l|l|}
\hline Link Gains & Coefficient Set 1 & Coefficient Set 2 \\
\hline \hline$s_{10}, s_{20}, s_{30}$ & $\{0.1: 0.2: 0.9\}$ & $\{0.5: 0.05: 0.7\}$ \\
$s_{13}, s_{21}, s_{32}$ & $\{1.1: 0.2: 1.9\}$ & $\{0.8: 0.05: 1.0\}$ \\
$s_{12}, s_{23}, s_{31}$ & $\{2.1: 0.2: 2.9\}$ & $\{1.1: 0.05: 1.3\}$ \\
\hline
\end{tabular}

TABLE V

FADING DISTRIBUTIONS SATISFYING (6)

\begin{tabular}{|l|l|l|}
\hline Link Gains & Coefficient Set 3 & Coefficient Set 4 \\
\hline \hline$s_{10}, s_{20}, s_{30}$ & $\{0.1: 0.2: 0.9\}$ & $\{0.5: 0.05: 0.7\}$ \\
$s_{13}, s_{23}, s_{31}$ & $\{1.1: 0.2: 1.9\}$ & $\{0.8: 0.05: 1.0\}$ \\
$s_{12}, s_{21}, s_{32}$ & $\{2.1: 0.2: 2.9\}$ & $\{1.1: 0.05: 1.3\}$ \\
\hline
\end{tabular}

two user cooperation case, due to the asymmetry in the interuser links. Therefore, the separately obtained 2-D achievable rate regions on each plane has different intersections with the corresponding axes. However, in three user cooperation, the presence of the third user helps the user with the worse cooperative link by presenting another option to relay its information, thereby symmetrizing the achievable rate region, and providing a fairer rate distribution.

We next compare the rate regions achievable by policies 1 and 2, under four different fading distributions; two of which obey (6), and the remaining two of which obey (8). The fading distributions are again chosen as independent uniform random variables, as summarized in Tables IV and V.

Figure 3 illustrates the rate regions achievable by policy I, policy II, and the two user cooperative MAC, under the assumption that the fading distributions obey (8). This is the ordering for which the three user cooperation policy II in this paper is designed. Therefore, it is expected that the proposed policy gives significantly larger achievable rates than the other policies, for the same channel set. It is worth mentioning that, the policy I of [1] performed surprisingly poorly when compared to the 2 user cooperation strategy, which is simply a special case of policy I encoding-wise. The reason for this phenomenon is that, the decoding rules are strictly dictated in policy $\mathrm{I}$, and we force user 2 , which has a relatively poor incoming link from user 3 , to decode all sub-messages. Therefore, even if it will not participate in the transmission, user 2 creates a bottleneck for the rate of user 3 . One last remark: the rate plane $R_{1}-R_{3}$ was chosen arbitrarily for the comparison, all other rate regions also look similar.

In Figure 4, two alternative sets of fading distributions, each of which obey (6) are considered. Surprisingly, especially for

$$
\begin{gathered}
R_{12}<E\left[\log \left(1+s_{10}\left(P_{12}+P_{1 U 1}\right)+s_{20}\left(P_{21}+P_{2 U 1}\right)+D\right)\right] \\
R_{31}<E\left[\log \left(1+s_{10}\left(P_{13}+P_{1 U 3}\right)+s_{30}\left(P_{31}+P_{3 U 3}\right)+E\right)\right] \\
R_{23}<E\left[\log \left(1+s_{20}\left(P_{23}+P_{2 U 2}\right)+s_{30}\left(P_{32}+P_{3 U 2}\right)+F\right)\right] \\
R_{12}+R_{23}<E\left[\log \left(1+s_{10}\left(P_{12}+P_{1 U 1}\right)+s_{20}\left(P_{21}+P_{23}+P_{2 U 1}+P_{2 U 2}\right)+s_{30}\left(P_{32}+P_{3 U 2}\right)+D+F\right)\right] \\
R_{12}+R_{31}<E\left[\log \left(1+s_{10}\left(P_{12}+P_{13}+P_{1 U 1}+P_{1 U 3}\right)+s_{20}\left(P_{21}+P_{2 U 1}\right)+s_{30}\left(P_{31}+P_{3 U 3}\right)+D+E\right)\right] \\
R_{23}+R_{31}<E\left[\log \left(1+s_{10}\left(P_{13}+P_{1 U 3}\right)+s_{20}\left(P_{23}+P_{2 U 2}\right)+s_{30}\left(P_{31}+P_{32}+P_{3 U 2}+P_{3 U 3}\right)+E+F\right)\right] \\
R_{12}+R_{23}+R_{31}<E\left[\operatorname { l o g } \left(1+s_{10}\left(P_{12}+P_{13}+P_{1 U 1}+P_{1 U 3}\right)+s_{20}\left(P_{21}+P_{23}+P_{2 U 1}+P_{2 U 2}\right)\right.\right. \\
\left.\left.\quad+s_{30}\left(P_{31}+P_{32}+P_{3 U 2}+P_{3 U 3}\right)+D+E+F\right)\right] \\
R_{1}+R_{2}+R_{3}<E\left[\log \left(1+s_{10} P_{1}+s_{20} P_{2}+s_{30} P_{3}+D+E+F+G\right)\right]
\end{gathered}
$$


coefficient set 3, our proposed policy II performs better than policy I, although it was not designed for the assumed ordering of the fading values. This shows that, enforcing user 2 to decode all messages, while treating most messages as noise at user 3 may be more limiting than letting each user decode an equal number of sub-messages, under certain situations. When the potential channel states get closer to each other, as in coefficient set 4, policy II partly outperforms policy I. Also, policy II outperforms the two user cooperative strategy under this ordering, as expected (decoding at user 2 is no longer a bottleneck).

\section{CONCLUSIONS}

In this paper, we have proposed a new block Markov type encoding strategy for the three user multiple access channel. The encoding and decoding policies were developed by making use of a specific ordering of the channel states, complementing our existing results [1], thereby yielding a complete channel adaptive encoding policy. We have obtained the rate constraints for reliable decoding of messages for the three user multiple access channel under our proposed encoding and decoding strategies, and evaluated them to obtain three user achievable rate regions. We demonstrated through simulations that, going from the two user cooperative multiple access channel to its three user counterpart, the achievable rates increase significantly due to the additional diversity provided by the existence of an extra user.

\section{REFERENCES}

[1] C. Edemen and O. Kaya Achievable Rates for the Three User Cooperative Multiple Access Channel. In Proc IEEE Wireless Communications and Networking Conference, Las Vegas, NV, March-April 2008.

[2] A. B. Carleial. Multiple Access Channels with Different Generalized Feedback Signals. IEEE Trans. on Inf. Theory, 28(6):841-850, Nov. 1982.

[3] F. M. J. Willems, E. C. van der Meulen and J. P. M. Schalkwijk. An Achievable Rate Region for the Multiple Access Channel with Generalized Feedback. In Proc. Allerton Conf., Monticello, IL, Oct. 1983.

[4] A. Sendonaris, E. Erkip and B. Aazhang. User Cooperation Diversity Part I: System Description. IEEE Trans. on Communications, 51(11): 1927-1938, Nov. 2003.

[5] G. Kramer, M. Gastpar amd P. Gupta. Cooperative Strategies and Capacity Theorems for Relay Networks. IEEE Trans. Inf. Theory, 51(9):3037-3063, Sept. 2005.

[6] P. Gupta and P. R. Kumar. Toward an information theory of large networks: An achievable rate region. IEEE Trans. Inf. Theory,49(8):18771894, Aug. 2003.

[7] B. Schein and R. G. Gallager. The Gaussian parallel relay network. In Proc IEEE Int. Symp. Inf. Theory, Sorrento, Italy, Jun. 2000, p. 22.

[8] G. Kramer, M. Gastpar, and P. Gupta. Capacity theorems for wireless relay channels. In Proc. Allerton Conf., Monticello, IL, Oct. 2003, pp. 1074-1083.

[9] L. L. Xie and P. R. Kumar. A network information theory for wireless communication: Scaling laws and optimal operation. IEEE Trans. Inf. Theory, 50(5):748-767, May 2004.

[10] L. L. Xie and P. R. Kumar. An achievable rate for the multiple-level relay channel. IEEE Trans. Inf. Theory, 51(4):13481358, Apr. 2005.

[11] L. Sankaranarayanan, G. Kramer and N. B. Mandayam. Capacity Theorems for the Multiple-Access Relay Channel In Proc. Allerton Conf., Monticello, IL, Sept./Oct. 2004.

[12] G. Kramer and A. J. van Wijngaarden. On the White Gaussian MultipleAccess Relay Channel In Proc IEEE Int. Symp. Inf. Theory, Sorrento, Italy, Jun. 2000, p. 40.

[13] O. Kaya and S. Ulukus. Power Control for Fading Cooperative Multiple Access Channels. IEEE Trans. on Wireless Communications, 6(8):29152923, August 2007.

[14] T. M. Cover and J. A. Thomas. Elements of Information Theory. Wiley Interscience, 1991.

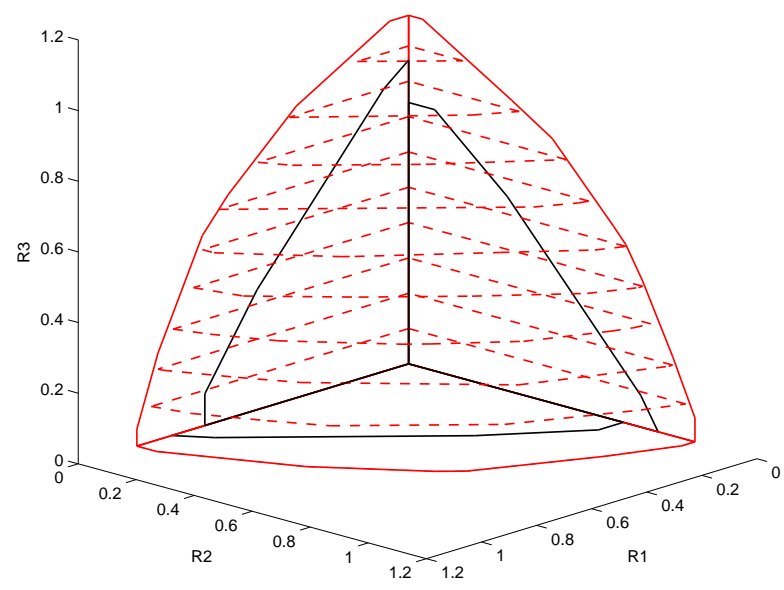

Fig. 2. The 3-D Achievable Rate Region for the three user cooperative MAC, compared with two user cooperative rate regions.

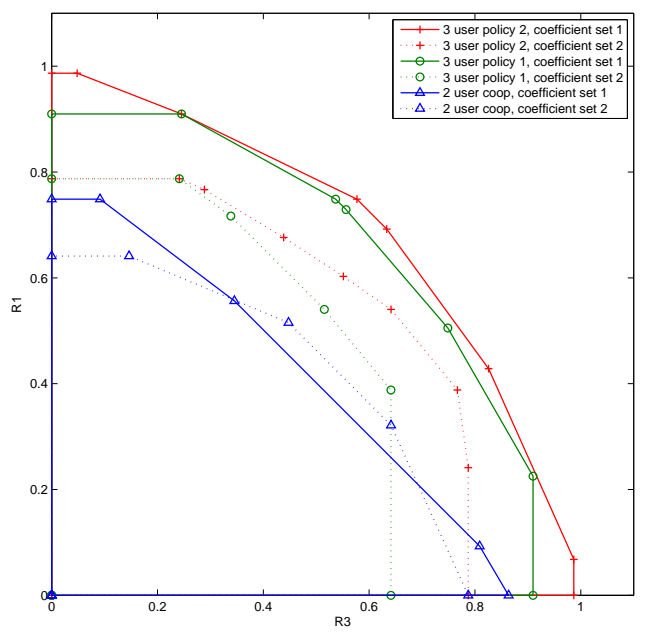

Fig. 3. Achievable rate regions for the cooperative MAC, coefficients obeying (8), $R_{2}=0$.

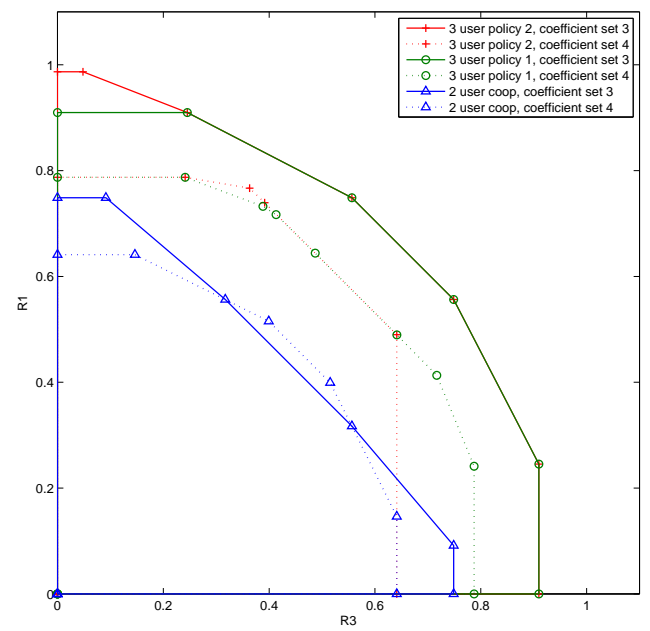

Fig. 4. Achievable rate regions for the cooperative MAC, coefficients obeying (6), $R_{2}=0$. 